\title{
The effect of iron supplementation on HBA1c levels in non-diabetic pregnant women.
}

\author{
Colak Eser ${ }^{1 *}$, Esinler Deniz ${ }^{2}$, Yerebasmaz Neslihan², Tohma Aytac ${ }^{1}$, Kandemir Omer ${ }^{2}$, Yalvac Serdar 3 \\ ${ }^{1}$ Baskent University Faculty of Medicine, Department of Obstetrics and Gynecology, Konya, Turkey \\ ${ }^{2}$ Etlik Zubeyde Hanim Maternity Hospital, Department of Obstetrics and Gynecology, Division of Maternal and Fetal \\ Medicine, Ankara, Turkey \\ ${ }^{3}$ Bozok University Faculty of Medicine, Department of Obstetrics and Gynecology, Yozgat, Turkey
}

\begin{abstract}
Background: It is estimated that $41,8 \%$ of pregnant women worldwide are anemic. At least half of this burden is assumed to be due to iron deficiency. Although iron deficiency anemia is very common in pregnancy, there has been limited research investigating glycosylated hemoglobin (HBA1c) levels in nondiabetic anaemic pregnant women and the effect of iron supplementation on HbA1c levels. We aimed to investigate HBA1c level changes in anemic pregnant women after anemia is corrected and to evaluate the relation of HBA1c with haemoglobin, haematocrit, ferritin and red blood cell indices.

Materials and methods: Thirty seven pregnant women (16-30 weeks into their pregnancies) who had been diagnosed as having iron deficiency anemia (IDA) were enrolled in the study. IDA was corrected with iron supplementation. Haemoglobin, haematocrit, ferritin and red blood cell indices were analysed prior to and following (1 month) iron replacement therapy.

Results: HBA1c values significantly decreased after iron supplementation when compared to those in a pre-supplementation state $(5.01 \pm 0.39$ vs. $4.69 \pm 0.38)$.

Conclusion: The correction of iron deficiency anemia (IDA) in pregnant women via iron supplementation significantly decreased HBA1c values. Thus, the interpretation of HBA1c values should be carefully conducted in the presence of IDA in the case of pregnancy. If there is IDA in pregnancy, it should be corrected prior to making any decisions based on HBA1c values.
\end{abstract}

Keywords: HBA1c, Iron deficiency anemia, Pregnancy.

Accepted on December 05, 2017

\section{Introduction}

Gycosylated hemoglobin (HBA1c) has a glucose residue attached to the terminal $\mathrm{NH} 2$ group (valine residue) of one or both $\mathrm{HbA}$ beta chains [1]. It is widely used by physicians to inquire past plasma glucose levels in last 2-3 months [1]. Several factors such as anemia, structural hemoglobinopathies and thalassemia syndromes may affect HBA1c results. Depending on the type of anemia, it may be associated with a rapid turnover of erythrocytes, resulting in lower HBA1c levels, or alternatively, slower turnover of erythrocytes, resulting in increased glycosylation of $\mathrm{Hb}$ and consecutively, higher HBA1c levels [2]. Accordingly, the presence of anemia may result in false interpretations of HBA1c results. In hemolytic anemia species and in patients with severe blood loss, there is a decrease in HbAlc levels and there is an increase in cases that lead to an increase in erythrocyte age such as iron deficiency anemia [2].

It is estimated that $41.8 \%$ of pregnant women worldwide are anemic [3]. At least half of this burden is assumed to be due to iron deficiency [3]. Although iron deficiency anemia is very common in pregnancy, to our best knowledge, there has been limited research investigating HBA1c levels in non-diabetic anaemic pregnant women and the effect of iron supplementation on HBA1c levels [4-6]. In order to see the effect of anemia treatment on HBA1c levels, we analysed HBA1c levels in non-diabetic pregnant women before and after iron supplementation.

\section{Materials and Methods}

The study was designed as prospective and was conducted at the Perinatology Department of Etlik Zubeyde Hanim Womens Health and Teaching Hospital. The Institutional Review Board approved the study. Thirty-seven pregnant women (between 16-30 weeks gestation) diagnosed with iron deficiency anemia (IDA) from June 2013 to February 2014, were enrolled in the study. In our study, iron-deficiency anemia was diagnosed when the hemoglobin level was below $11 \mathrm{gr} / \mathrm{dL}$ in the first and third trimesters or below $10.5 \mathrm{gr} / \mathrm{dL}$ in the second trimester; 
mean cell volume was lower than $75 \mathrm{fl}$ and the serum ferritin level was lower than $30 \mathrm{ng} / \mathrm{mL}$ in the absence of the other causes of anemia. Pregnant patients with hemoglobin levels of 6-11 gr/dL were included in the study if the anemia was iron deficiency anemia and the patient was either intolerant or did not respond to oral iron therapy despite regular use. Patients having iron-deficiency anemia with hemoglobin levels below 6 $\mathrm{gr} / \mathrm{dL}$ or symptomatic (severe fatigue, palpitations, tachycardia, shortness of breath) patients with hemoglobin levels greater than $6 \mathrm{gr} / \mathrm{dL}$ were candidates for RBC transfusion and those were excluded. After informed consent for intravenous iron therapy was taken, the patients were hospitalized. Patients with fasting plasma glucose levels $>110 \mathrm{mg} / \mathrm{dL}$ and 50 gram glucose challenge test $>140 \mathrm{mg} / \mathrm{dL}$ as well as patients with hemoglobinopathy, gestational diabetes mellitus (GDM) or Type I-II diabetes mellitus were excluded from the study were excluded from the study. The iron sucrose dosage was calculated according to the following formula:

Total iron deficit $[\mathrm{mg}]=$ body weight $[\mathrm{kg}] \times[$ target $\mathrm{Hb}$ (usually taken as $11 \mathrm{gr} / \mathrm{dL}$ )-actual $\mathrm{Hb}][\mathrm{g} / \mathrm{dL}] \times 2.4+$ depot iron (usually taken as 500) [mg] [7].

The total amount of iron sucrose to be administered (in $\mathrm{mL}$ ) was calculated by total iron deficit (mg) divided by $20 \mathrm{mg} / \mathrm{mL}$. Iron sucrose was diluted only in sterile $0.9 \%$ sodium chloride solution as $100 \mathrm{mg}$ iron ( $5 \mathrm{~mL}$ iron sucrose) in maximum 100 $\mathrm{ml}$ sterile $0.9 \%$ sodium chloride solution and for stability reasons, dilutions to lower iron sucrose concentrations was not allowed. As infusion, maximum tolerated single dose per day given, not more than once per week was: $500 \mathrm{mg}$ iron $(25 \mathrm{~mL}$ iron sucrose) in at least $3 \mathrm{~h}$ for patients above $70 \mathrm{~kg}$ and $7 \mathrm{mg}$ iron $/ \mathrm{kg}$ body weight in at least $3 \mathrm{~h}$ for patients of $70 \mathrm{~kg}$ and below. If the total dose to be administered was greater than the maximum tolerated single dose per day, the remaining dose was administered one week later. Hemoglobin $(\mathrm{Hb})$, hematocrit (Htc), mean corpuscular volume (MCV), mean corpuscular hemoglobin $(\mathrm{MCH})$, mean corpuscular hemoglobin concentration (MCHC), ferritin and HBAlc levels were measured before and at the end of the first month following iron supplementation. Direct enzymatic HBA1c assay (Abbott Laboratories, IL, USA) with intra- and inter percentage CV values of less than $3 \%$ was used to measure HBA1c values. Statistical analyses were performed using the SPSS version 21.0 (SPSS, Chicago, IL). Paired sample student t-test was used to compare HBA1c level changes. Pearson correlation coefficient was used to analyse the degree of linear dependence between two variables. Statistical tests were considered significant at the $95 \%$ level.

\section{Results}

Of 37 women enrolled into the study, $29.7 \%$ were primigravida and others were multigravida (second pregnancy in $32.4 \%$, third pregnancy in $21.6 \%$, 4th pregnancy in 10.8 , 5th pregnancy in $2.7 \%$ and 7 th pregnancy in $2.7 \%$; Table 1 ). Mean fasting blood glucose (FBG) is $81.11 \pm 11.14$, while mean glucose challenge test $50 \mathrm{~g}$ is $103.73 \pm 17.05$. Median value of body mass index (BMI) is 35 , and median value is 25 weeks by gestational age (Table 1). As expected, following iron supplementation, $\mathrm{Hb}, \mathrm{Htc}, \mathrm{MCV}, \mathrm{MCH}, \mathrm{MCHC}$ and ferritin levels were significantly increased compared to presupplementation levels (Table 2). However, HBA1c levels were significantly lower following iron supplementation (5.01 $\pm 0.39 v s .4 .69 \pm 0.38, \mathrm{p}<0.05)$ (Table 2). We failed to find any significant correlation between the degree of HBA1c changes and the changes in parameters $\mathrm{Hb}, \mathrm{Htc}, \mathrm{MCV}, \mathrm{MCH}, \mathrm{MCHC}$ and ferritin.

Table 1. Basal characteristics of patients included in the study $(n=37)$.

\begin{tabular}{ll}
\hline Variable & $\mathrm{n}=37$ \\
\hline Gestational age $($ week $)$ & $25.0 \pm 2.5$ \\
\hline Body mass index $\left(\mathrm{kg} / \mathrm{m}^{2}\right)$ & $26.2 \pm 3.9$ \\
\hline Fasting plasma glucose $(\mathrm{gr} / \mathrm{dL})$ & $81.1 \pm 11.4$ \\
\hline 50 gr oral glucose tolerance test $(\mathrm{mg} / \mathrm{dL})$ & $103.7 \pm 17.0$ \\
\hline
\end{tabular}

Table 2. Comparison of glycosylated haemoglobin (HbAlc), hemoglobin, hematocrit, ferritin and red blood cell indices before iron supplementation and post-iron supplementation.

\begin{tabular}{|c|c|c|c|}
\hline Variable & $\begin{array}{l}\text { Pre-iron } \\
\text { supplementatio } \\
\mathbf{n}\end{array}$ & $\begin{array}{l}\text { Post-iron } \\
\text { supplementation }\end{array}$ & P-value \\
\hline $\mathrm{HbA} 1 \mathrm{c}(\%)$ & $5.01 \pm 0.39$ & $4.69 \pm 0.38$ & $p<0.01$ \\
\hline Hemoglobin (gr/dL) & $9.8 \pm 0.9$ & $10.6 \pm 1.0$ & $p<0.01$ \\
\hline Hematocrit (\%) & $25.2 \pm 2.5$ & $32.3 \pm 3.1$ & $p<0.01$ \\
\hline Ferritin $(\mathrm{ng} / \mathrm{mL})$ & $6.3 \pm 2.1$ & $37.6 \pm 6.7$ & $p<0.01$ \\
\hline $\begin{array}{l}\text { Mean corpuscular volume } \\
(\mathrm{fL})\end{array}$ & $71.2 \pm 8.1$ & $82.0 \pm 7.8$ & $p<0.01$ \\
\hline $\begin{array}{l}\text { Mean corpuscular } \\
\text { hemoglobin (pg/cell) }\end{array}$ & $22.7 \pm 3.4$ & $26.7 \pm 2.9$ & $p<0.01$ \\
\hline $\begin{array}{l}\text { Mean } \\
\text { haemoglobin corpuscular } \\
(\mathrm{g} / \mathrm{dL})\end{array}$ & $31.7 \pm 1.3$ & $32.5 \pm 1.1$ & $p<0.05$ \\
\hline
\end{tabular}

$\mathrm{HbA} 1 \mathrm{c}=$ glycosylated hemoglobin; ferritin was given as a mean \pm standard error

\section{Discussion}

Iron therapy is the mainstay of iron-deficiency anemia treatment and intravenous iron is increasingly used when oraltherapy is not effective or well tolerated. In this study, we found that intravenous iron therapy is effective in iron restoration and anemia treatment with few changes on laboratory values. Worth et al. investigated changes in HBA1c values in 21 pregnant women and showed that HBA1c reached at its nadir at 17 weeks, peaked at delivery, and dropped in the postpartum period [8]. Similarly, Phelps et al. analysed HBA1c concentrations throughout gestation in 377 non-diabetic pregnant women [9]. They noted significant biphasic changes in HBA1c concentrations, with an initial gradual decline to nadir level at 24 weeks, followed by a subsequent slow reascension to peak near term. Both authors concluded these changes in HBA1c as physiological changes related to 
pregnancy and that care should be taken during the interpretation of HBAlc results in the presence of pregnancy. However, they did not suggest any possible mechanism for the cause(s) of these changes.

The effect of iron deficiency anemia (IDA) on HBA1c values was first investigated by Horton and Huisman [10], who noted the mean $\mathrm{HbA} 1 \mathrm{c}$ concentration for four patients with IDA as $4.9 \%$, compared to a mean HBA1c concentration of $5.3 \%$ among 14 healthy adults. In 1980, on the contrary, Brooks et al. [11] investigated HBA1c levels in 25 non-diabetic patients with IDA both prior to and following treatment with $1 \mathrm{v}$ iron supplementation for six weeks and they reported that the mean HBA1c values were significantly decreased following iron supplementation (9.9 \pm 0.3 vs. $7.9 \pm 0.13$, respectively) and that IDA was associated with higher HBA1c values.

Tarim et al. [12] reported that patients $(n=11)$ with Type I diabetes mellitus (DM) and IDA had higher levels of HBA1c than patients with DM but without IDA $(n=26)(10.6 \pm 2.6 v s$. $7.7 \pm 1.3$, respectively). In their study, iron supplementation significantly decreased mean HBA1c values in patients with $\mathrm{DM}(10.1 \pm 2.7$ vs. $8.2 \pm 3.1)$. In accordance to the results of our study, Tarim et al. additionally noted that iron supplementation also decreased HBA1c levels in non-diabetic patients with IDA $(7.6 \pm 2.6 \%$ vs. $6.2 \pm 1.4)$. El-Agouza et al. [13] investigated the effect of iron deficiency anemia on the levels of HBA1c in 41 patients with IDA and noted that HBA1c fell significantly following iron supplementation from a mean of $6.15 \pm 0.62$ to $5.25 \pm 0.45$. They also concluded that iron deficiency must be corrected before making any diagnostic or therapeutic decisions based on HBA1c levels. Coban et al. [14] investigated the effect of IDA on HBA1c values in 50 patients with IDA and 50 healthy control patients. HBA1c values in IDA patients were significantly higher compared to control patients prior to iron supplementation (7.4 \pm 0.8 vs. $5.2 \pm 0.2$, respectively). Also in accordance to our study, following iron supplementation, the HBA1c value in patients with IDA decreased significantly $(7.4 \pm 0.8$ vs. $6.2 \pm$ $0.6)$.

Koga et al. [15] demonstrated that red blood cell count was positively and hemoglobin, $\mathrm{MCH}$ and $\mathrm{MCV}$ were negatively associated with HBA1c levels in premenopausal women. Among the erythrocyte indices, the highest correlation coefficient for an association with HBA1c in pre-menopausal women was for $\mathrm{MCH}$. A decrease of $1 \mathrm{pg}$ in $\mathrm{MCH}$ corresponds to an increase of approximately $0.03 \%$ in HBA1c. Premenopausal women with low $\mathrm{MCH}(<27 \mathrm{pg})$ had significantly higher HBA1c levels. Koge et al. therefore concluded that the relatively iron deficient state of premenopausal women with lower $\mathrm{MCH}$ levels may be responsible for higher HBA1c levels. Recently, English et al. [4] reported a systematic review of 12 articles related to association of IDA on HBA1c levels and concluded that HBA1c was likely to be affected by iron deficiency and IDA, with a deceptive increase in HBA1c values. They also noted that this may lead to confusion when diagnosing diabetes using HBA1c.
In the literature, there is limited research investigating the relationship between IDA and HBA1c in pregnancy. Hashimoto et al. [5] conducted a study on 47 pregnant women in Japan in 2007 and found that HBA1c was negatively correlated with $\mathrm{MCH}$, serum transferrin saturation and serum transferrin. They concluded that an increase in HBA1c levels during late pregnancy appears primarily attributable to an irondeficient status at this period. Rafat et al. [6] recently assessed the influence of iron metabolism indices on HBA1c in nondiabetic pregnant women. They reported that HBA1c levels were higher in patients with IDA compared to controls. Moreover, HBA1c levels decreased from $5.2 \pm 0.3$ to $5.1 \pm 0.3$ following iron supplementation. They also observed a significant correlation between erythrocyte indices, iron metabolic indices and HBA1c levels.

In our study, we found that iron supplementation in patients with IDA significantly decreased HBA1c values. However, we failed to find a significant correlation between the degree of HBA1c change and the change in parameters $\mathrm{Hb}, \mathrm{Htc}, \mathrm{MCV}$, $\mathrm{MCH}, \mathrm{MCHC}$ and ferritin. The reason for this may be the small sample size. There are different hypotheses for explaining HBA1c increments in patients with IDA. Sluiter et al. [16] hypothesized that the formation of glycosylated hemoglobin was an irreversible process and therefore, the concentration of HBA1c in one erythrocyte would increase linearly according to the cell's age. However, in the case of an iron deficiency, red cell production rate would have dropped, leading not only to anemia but also to a higher than normal average age of circulating erythrocytes and therefore, increased HBA1c. Following IDA correction with iron supplementation, many younger erythrocytes will enter circulation, which in turn will result in a significant decrease in HBA1c levels. From our point of view, this hypothesis is the most instructive and logical option.

In the literature, there are a limited number of studies reporting that HBA1c values do not change in the presence of IDA. In a study conducted by Heyningen et al. [17], authors reported no statistically significant differences in HBA1c values, which contradict our study and those in the literature. Likewise, Rai et al. [18] reported no differences between the HBA1c levels of 12 control subjects and 15 patients with IDA.

\section{Conclusions}

In our study the correction of iron deficiency anemia (IDA) in pregnant women by iron supplementation significantly decreased HBA1c values. Thus, IDA should be taken into account in the interpretation of HBA1c values in pregnancy.

\section{References}

1. Sacks DB, Arnold M, Bakris GL, Bruns DE, Horvath AR, Kirkman MS. Guidelines and recommendations for laboratory analysis in the diagnosis and management of diabetes mellitus. Clin Chem 2011; 57: 1-47.

2. Ahmad J, Rafat D. Hba1c and iron deficiency: A review. Diabetes Metabol Syndrome 2013; 7: 118-122. 
3. McLean E, Cogswell M, Egli I, Wojdyla D, Benoist B. Worldwide prevalence of anemia, who vitamin and mineral nutrition information system, 1993-2005. Public Health Nutrition 2009; 12: 444-454.

4. English E, Idris I, Smith G, Dhatariya K, Kilpatrick ES, John WG. The effect of anemia and abnormalities of erythrocyte indices on hbalc analysis: A systematic review. Diabetologia 2015; 58: 1409-1421.

5. Hashimoto K, Osugi T, Noguchi S, Morimoto Y, Wasada K, Imai S. A1c but not serum glycated albumin is elevated because of iron deficiency in late pregnancy in diabetic women. Diabetes Care 2010; 33: 509-511.

6. Rafat D, Rabbani TK, Ahmad J, Ansari MA. Influence of iron metabolism indices on hbalc in non-diabetic pregnant women with and without iron-deficiency anemia: Effect of iron supplementation. Diabetes Metabolic Syndrome 2012; 6: 102-105.

7. http://www.medsafe.govt.nz/profs/Datasheet/f/ FerrumHinj.pdf

8. Worth R, Potter JM, Drury J, Fraser RB, Cullen DR. Glycosylated haemoglobin in normal pregnancy: A longitudinal study with two independent methods. Diabetologia 1985; 28: 76-79.

9. Phelps RL, Honig GR, Green D, Metzger BE, Frederiksen MC, Freinkel N. Biphasic changes in hemoglobin alc concentrations during normal human pregnancy. Am J Obstet Gynecol 1983; 147: 651-653.

10. Horton BF, Huisman TH. Studies on the heterogeneity of haemoglobin. Vii. Minor haemoglobin components in haematological diseases. Br J Haematol 1965; 11: 296-304.

11. Brooks AP, Metcalfe J, Day JL, Edwards MS. Iron deficiency and glycosylated haemoglobin a. Lancet 1980; 2: 141 .
12. Tarim O, Kucukerdogan A, Gunay U, Eralp O, Ercan I. Effects of iron deficiency anemia on hemoglobin alc in type 1 diabetes mellitus. Pediatrics Int 1999; 41: 357-362.

13. El-Agouza I, Abu Shahla A, Sirdah M. The effect of iron deficiency anemia on the levels of haemoglobin subtypes: Possible consequences for clinical diagnosis. Clin Lab Haematol 2002; 24: 285-289.

14. Coban E, Ozdogan M, Timuragaoglu A. Effect of iron deficiency anemia on the levels of hemoglobin a1c in nondiabetic patients. Acta Haematologica 2004; 112: 126-128.

15. Koga M, Morita S, Saito H, Mukai M, Kasayama S. Association of erythrocyte indices with glycated haemoglobin in pre-menopausal women. Diabetic Med 2007; 24: 843-847.

16. Sluiter WJ, van Essen LH, Reitsma WD, Doorenbos H. Glycosylated haemoglobin and iron deficiency. Lancet 1980; 2: 531-532.

17. Van Heyningen C, Dalton RG. Glycosylated haemoglobin in iron-deficiency anemia. Lancet 1985; 1: 874.

18. Rai KB, Pattabiraman TN. Glycosylated haemoglobin levels in iron deficiency anemia. Indian J Med Res 1986; 83: 234-236.

\section{*Correspondence to}

Colak Eser

Baskent University Faculty of Medicine

Department of Obstetrics and Gynecology

Turkey 